\title{
Energy-based Method to Simplify Complex Multi-Energy Modelica Models
}

\author{
Joy El Feghali ${ }^{1} \quad$ Guillaume Sandou $^{1}$ Hervé Guéguen ${ }^{2} \quad$ Pierre Haessig $^{2}$ Damien Faille ${ }^{3}$ \\ ${ }^{1}$ Université Paris-Saclay, CNRS, CentraleSupélec, Laboratoire des signaux et systèmes, 91190, Gif-sur-Yvette, \\ France, $\{$ joy.el-feghali, guillaume.sandou\}@centralesupelec.fr \\ ${ }^{2}$ IETR, CentraleSupélec, Rennes, France, \{herve.gueguen, pierre.haessig\} @centralesupelec.fr \\ ${ }^{3}$ Electricité de France, R\&D, PRISME Department, 78400, Chatou, France, damien. faille@edf. fr
}

\begin{abstract}
Energy production and consumption systems increasingly require more flexibility. The design of new control solutions can be a step, among others, towards flexibility. However, these control solutions often rely on the use of complex models, which are difficult to both manipulate and simulate. This paper presents a proof of concept of a method that reduces the complexity of multi-energy models modeled with Modelica language. This complexityreducing method is based on simplifying the model's components that contribute less to the total energy using an energy-based ranking technique. The proposed solution is successfully applied to a complex city district model. A property of the Modelica language further allows redeclaration of low-ranked components without being compelled to fully redesign the model. Criteria verifying the multi-energy reduced model's precision, while respecting physical constraints, are also introduced.

Keywords: energy-based ranking, model reduction, multienergy systems, Modelica
\end{abstract}

\section{Introduction}

A significant research effort is required in energy production to reduce fossil energy use and move towards a sizeable renewable energy penetration. Alongside this, optimizing energy consumption at the utility level (residential, tertiary, and industrial sectors) can increase global energy efficiency and decrease energy needs. The energy transition thus requires greater flexibility both on the production and consumption sides. This need has to be supported by new control systems. For this purpose, dynamical models are needed for control design.

The Modelica language (Fritzson and Engelson 1998) has been chosen as the modeling framework for dynamical models, thanks to its ability to capture multi-physics systems. However, this physical modeling may lead to complex or even intractable models, which cannot be used for control purposes and control law design. As a result, a suitable model obtained from a reduction of the full physical-based model is often necessary. This paper aims to provide a methodology to reduce the model's complexity by using a reduction technique applied to Modelica models.

In the model reduction literature, conventional methods are widely used, such as modal truncation method (Marshall 1966) and balanced truncation or Moore's method (Moore 1981). The modal truncation method aims to separate slow dynamics and fast dynamics in a modal base, while eliminating fast dynamics that influence the system less. With Moore's method, less controllable and less observable states are eliminated in a balanced base. These methods also require a linear system where eigenvalues and singular values are identified. However, physical models used for energy systems are not always linear, in which case energy models need to be linearized before applying reduction methods. This approach was used for Modelica models in Kim et al. (2014), building models were simplified in Modelica using physical properties and then reduced using Moore's method. Since this method is dedicated to LTI models, the physically simplified model of the building in Modelica was linearized before using the reduction method in Matlab. Although interesting for some specific components such as buildings, these methods appear hard to be generalized in the case of multienergy systems due to the versatility of the non-linearities that need to be taken into account and the different operating conditions that should be considered.

Another drawback of these methods comes with the loss of the model structure. The models are indeed often obtained thanks to a change of basis in the model variables with difficult-to-interpret physical meaning. Methods, like the aggregation of states, allow preserving the structure of the model. Deng et al. (2014) applied this method to a nonlinear building model. An analogy is used between the linear dynamics part and a continuous-time Markov chain to apply a Markov chain aggregation method. For Modelica models, additional work should be done to verify if the model is compatible with a particular state representation form before applying the method.

With the goal of maintaining the model's structure, Sodja, Škrjanc, and Zupančič (2019) and Sodja, Škrjanc, and Zupančič (2020) use an energy-based method applied to Modelica models, presenting reduction techniques for differential-algebraic equations (DAE) implemented in Modelica and reduction techniques for object diagrams. 
The method is based on ranking procedures that help to eliminate equations or components with less of an influence over models. The methodology seems to be extendable to multi-energy systems and particularly well suited to the Modelica framework. Thus, this paper is based on the same approach used in Sodja, Škrjanc, and Zupančič (2019), where components are ranked according to their energy contribution to the system. The components that contribute less to the total energy of the system are simplified. This energy-based ranking method can be applied to Modelica language models after some adaptation to ease the energy flow calculation in the multi-energy system components. In this paper, the ranking-procedure method is applied on a multi-energy system benchmark provided by our industrial partner. The low-ranked components will be automatically replaced using a Modelica property. Criteria for local and global error calculation are introduced to maintain a good precision of the reduced model while respecting the physical constraints.

In Section 2, the energy-based ranking method used in Sodja, Škrjanc, and Zupančič (2019) will be detailed. In Section 3, a solution to reduce the model with simple user intervention is presented. Particularities of multi-energy models are highlighted, and the city district model used to test the simplification method is introduced in Section 4. In Section 5, the numerical results both from the simulation times and representativeness points of view are presented. In Section 6, additional perspectives are proposed.

\section{Energy-based ranking method}

The energy-based ranking method ranks the components of a model according to an energy metric. Initially introduced for bond graphs, this method was extended in Sodja, Škrjanc, and Zupančič (2019) to reduce Modelica models due to the similarity of the structure between the objectoriented models in Modelica and the bond graphs. While Modelica models can contain flow and effort variables, their product does not always correspond to the energy flow. Further modifications in the model are thus needed to calculate the energy flow. A metric is then chosen to rank the components of the model. Low-ranked components will be replaced with simplified component models.

\subsection{Energy-flow calculation}

All connections to a component $i$ should be identified to calculate the energy flow in Modelica. The sum of all energy flows exchanged with other components $k$ gives the total energy flow of a component $i$ as defined in Equation 1.

$$
\dot{E}_{i}(t)=-\sum_{k} \dot{E}_{i, k}(t)
$$

The energy flow variable of each component defined in Equation 1 may not be directly available in the models and should be calculated by adding equations for energy flow calculation. Each Modelica component has a connector that links with a neighbor component $k$, and the information transferred through connectors helps to calculate the energy flowing to the component $i$. For example, with an electric pin connector of Modelica Standard Library, the voltage $v$ and the current $i$ are available. The product of these two variables gives the energy flow at the pin $\dot{E}=v * i$. Another example is a heat port connector of Modelica standard library; the temperature $T$ and the heat flow rate $Q_{\text {flow }}$ are available. The energy flow at the port is $\dot{E}=Q_{\text {flow }}$. Further examples of mechanics and fluid connectors can be found in Sodja, Škrjanc, and Zupančič (2019).

The energy flow is calculated during the simulation of the full model, after which the energy metric is calculated. For that purpose, a suitable energy metric should be chosen first.

\subsection{Activity Calculation}

Several metrics were introduced in (Sodja, Škrjanc, and Zupančič 2019), and the activity metric was chosen because it gives an idea of the error that the reduction causes. Initially, the activity was introduced in Louca (1998) for bond graphs. Activity of the component $i$, as defined in Equation 2, describes the energy flowing to a component $i$ during a time interval $\left[t_{1}, t_{2}\right]$.

$$
A_{i}=\int_{t_{1}}^{t_{2}}\left|\dot{E}_{i}(t)\right| d t
$$

Each component that stores or dissipates energy will have an activity value; this helps to rank the components relative to their energy contribution to the system. The reduction idea is to simplify the components ranked at the bottom due to their low energy contribution. In the case of eliminating a component, its contribution is removed, causing a difference between the full and the reduced models. The activity ranking thus gives the error value from a global perspective.

Notice that the activity ranking is not absolute but depends on the simulation inputs and the time interval. Indeed, the activity integral in Equation 2 is computed on a given simulation duration with particular input signals. In the car suspension example in Sodja, Škrjanc, and Zupančič (2019), it is shown that a high-frequency road profile input (i.e., sharp edges) yields the elimination of slow-moving components and vice versa.

\section{Modelica implementation of the re- placeable components}

A key question is how to remove a low-ranked component, or more precisely by which simplified model it should be replaced. The removal of a component leads to removing all of its connections. In Sodja, Škrjanc, and Zupančič (2019), the low-ranked components were removed manually by the user, and some modifications were needed to be done to avoid initialization problems. With large complex models, manual manipulation of the components may not be the best solution. Automatic modifications are 
Listing 1. Example of the replaceable Envelope

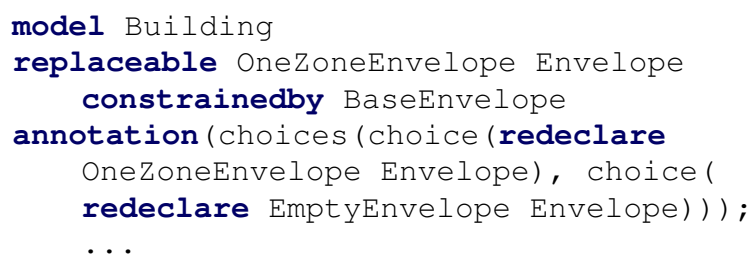

thus needed. In this paper, the authors propose replacing the low-ranked component with a simplified compatible component model with a minimum user intervention. A simplified component model can be created with the same connections to other components as the full component model but without all of its equations that bring complexity to the model. These equations could be removed, linearized, or replaced depending on the physics of the component. Suppose low-ranked components are replaced with empty component models, where all internal connections and equations are removed. In that case, the energy-based component's contribution is set to zero or the maximum or the mean energy value. Several compatible models can be defined in order to replace low-ranked components. The replacement choice will be left to the user, depending on the study.

Modelica has properties that help to replace a component with another compatible component model without having to redesign the system. Suppose a model Building that contains an Envelope component and an EnergySystem component. At the upper-level of the model Building, the Envelope will be replaced with another simplified model by choosing from a list of predefined components.

The following Modelica keywords replaceable, constrainedby, choices and redeclare help replacing the components (Tiller 2014). When components are created in the full model, the keyword "replaceable" is added to the component declaration to address a property to the component that it can change its type.

The keyword "constrainedby" is used to specify a constraining type for all the new compatible types by which the component can be replaced. The constrained type can be a base component model that compatible models inherit from.

In order to create a list of component types, new type choices are added to the annotation of the replaceable component in the full model. The choices determine the component model types' possibilities for replacement. The example of the replaceable Envelope's declaration in the model is given in Listing 1. At the upper-level of the complete model, using the keyword "redeclare", the replaceable component changes its type. The user can choose from a list of predefined component models and replace the original component model. The expression with "redeclare" is automatically added to the declaration of the component in the Modelica model, at the upperlevel, when choosing from a list. An example of the text
Listing 2. Example with redeclare at the upper-level of the model Building

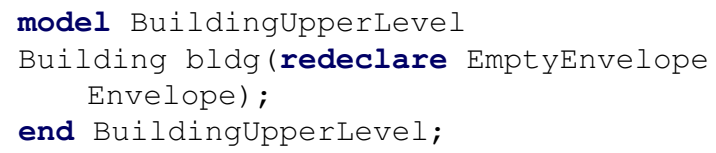

in Modelica for the Envelope redeclare at the upper level is shown in Listing 2.

\section{Multi-energy system case-study}

In this paper, the interest of the authors is to reduce the complexity of multi-energy models in Modelica by applying the energy-based reduction method. Multi-energy districts are considered, combining different types of energy production and consumption. These multi-energy districts are formed of buildings interacting with an electrical grid and a district heating network. The two latter systems can interact by a combined heat and power unit or by the electrical consumption of the district heating network. Renewable energy systems can also be connected.

The energy-based reduction method can be applied to these district Modelica models by choosing buildings as components to be ranked. Since buildings interact with different types of energy systems, they will have multiple energy flow variables. The energy flowing to a building is the power demanded from each of the connected energy systems. With a district composed of buildings communicating their power demands while interacting with an electrical grid and a district heating network, each building will have three types of activities: the active electrical activity $A_{P_{\text {elec }}}$, the reactive electrical activity $A_{Q_{\text {elec }}}$ and the heating activity $A_{P_{\text {heat }}}$. These variables correspond to the different energies flowing to the building for a chosen interval. Due to the multi-energy aspect of the system, these variables are separated.

After applying the energy-based ranking, the low-ranked buildings will be replaced with a simplified buildings model. At the source level, the error between the full and reduced models can be determined with the activity ranking. However, due to the interaction of the buildings with other systems like the electrical grid and heating network, removing a building causes error from a local perspective in the electrical grid model and heating network model. The precision of the reduced model should be checked at all levels, and the physical constraints of the model should be respected. For example, physical constraints for the electrical grid are checked for the voltage and the current values of the lines. The voltage should stay within a range of $\pm 5 \%$ of the nominal medium voltage for medium voltage lines and $\pm 10 \%$ of the nominal low voltage for low voltage lines. The current value should not exceed the $I_{\max }$ of the line.

Criteria of signals in the electrical grid and district heating network models are used to validate the reduced model de- 
pending on the system's physics. Criteria help to analyze how the signal is affected by the removal of the component.

The principles of the reduction of multi-energy models are applied in the case of a city district provided by Électricité de France (EDF R\&D). The system is modeled using EDF $\mathrm{R} \& \mathrm{D}$ Modelica libraries.

\subsection{Description of the model}

The chosen system is a sizeable multi-energy district located in the southern suburbs of Paris described by the PowerGrid demonstrator (Bouquerel et al. 2019). The district is composed of 719 buildings, one electrical grid, and one district heating network. The district's model in Dymola is complex due to its scale, and the computation time is thus long, raising the need for a reduction method. A smaller use case of 20 buildings is issued from the PowerGrid model to test the energy-based reduction method before applying it to a larger-scale model. These 20 buildings are connected to an electrical grid, and 12 of them are also connected to a district heating network. The buildings are local producers; they produce energy through photovoltaic panels (PV panels). The district heating network comprises a heat pump assisted by a storage tank that delivers hot water through pipes. EDF R\&D Modelica libraries are used to model the system in Dymola: BuildSysPro (Plessis, Kaemmerlen, and Lindsay 2014) for buildings, PowerSysPro (Tavella 2020) for the electrical grid, and MixSysPro, an internal EDF R\&D library for the district heating network.

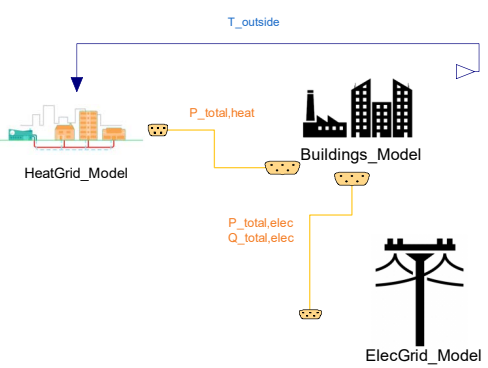

Figure 1. The multi-energy model in Dymola

The upper-level of the district model is composed of the buildings model, the electrical grid model, and the heating network model as shown in Figure 1.

In the buildings model as shown in Figure 2, the system is composed of an envelope model and an energy-system model. The envelope model calculates the interior temperature that is used in the energy-system model. In the energy-system model, the electrical power and heating power demands are calculated according to the consumption scenario. Buildings have solar panels, so they produce electrical power that the building will consume. If the PV production is higher than the need, the rest will be delivered to the electrical grid. The outputs of the buildings' model are the power demanded from the heating network and electrical grid. Each building connected to the electrical grid share its active $P_{\text {total,elec }}(t)$ and reactive electrical power $Q_{\text {total.elec }}(t)$. These electrical powers add all the electrical consumption demands of a building while reducing the amount produced locally by the PV panels. Each building connected to the heating network share its total heating consumption $P_{\text {total }}$,eat $(t)$. The heating power includes the hot water demand and the heater demand.

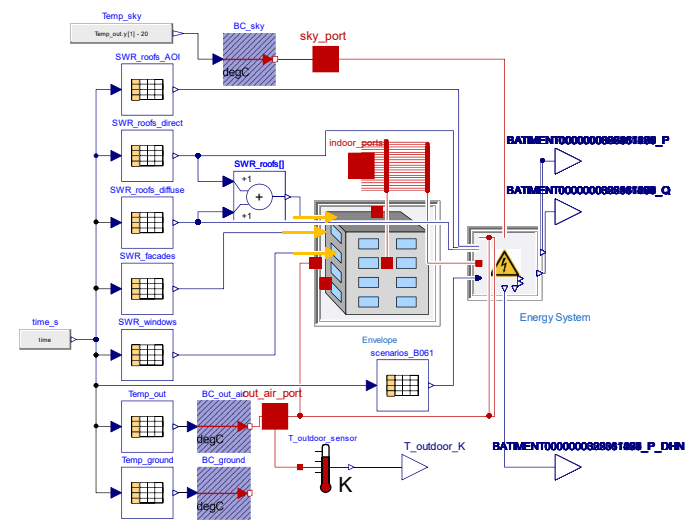

Figure 2. Buildings model in Dymola

The electrical grid model is composed of 23 lines. The 20 buildings are connected to low voltage or medium voltage lines as shown in Figure 3. The inputs of the electrical grid model are the electrical power demands of each building. The current and voltage of the lines are calculated within the model with power flow calculation.

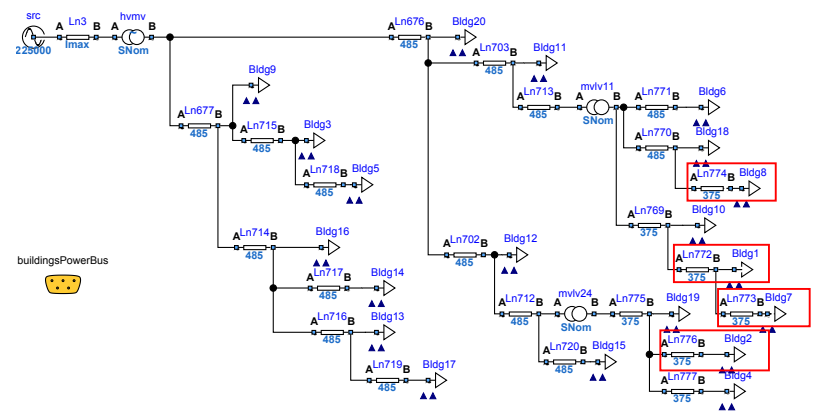

Figure 3. Electrical grid model in Dymola. The highlighted framed buildings are the subject of the subsection 5.2

The heating network model in Figure 4 contains the heat pump and the storage tank that feed the 12 substations connected to the 12 buildings. The buildings are assigned to one of the three subnetworks. The red and blue connections correspond to the hot water and cold water circulations, respectively. The inputs of the heating network model are the heating power demands of each 
building. The substations' return temperature $T_{\text {return }}(t)$ is calculated in the heating network model and depends only on the outside temperature. All buildings thus have the same return temperature $T_{\text {return }}(t)$. The mass flow is calculated for a building $i$ connected to a substation $i$ : $\dot{m}_{i}(t)=\frac{P_{\text {total }_{\text {heat }}}(t)}{c_{p}\left(T_{\text {supply }_{i}}(t)-T_{\text {return }}(t)\right)}$ with $T_{\text {supply }_{i}}(t)$ the temperature received at the substation $i$ and $c_{p}$ the fluid specific heat capacity.

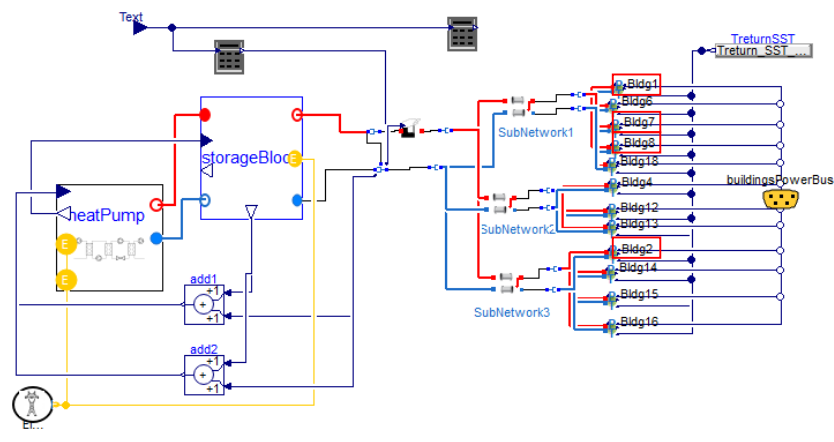

Figure 4. District heating network model in Dymola. The highlighted framed buildings are the subject of the subsection 5.2

\subsection{Impact of the reduction on the multi- energy model}

The simplification of the low-energy buildings affects both the electrical grid and district heating network models from global and local perspectives.

For the electrical grid, the global level corresponds to the electrical source level. The local level corresponds to the medium voltage and low voltage lines. Power, voltage, and current values of the lines will change in the electrical grid model due to the building simplification. The electrical power at the source level will vary slightly when removing a building with low energy contribution. However, at the line directly connected to the building removed, there will be a $100 \%$ error of the power, but which is supposed to be non-significant for the rest of the grid. The power error can be deduced from the activity ranking and the lines' position according to the buildings removed. The error of the current and voltage of the lines should be calculated by simulating the reduced model and comparing values with the full model. The voltage at a line is not allowed to vary significantly compared to the nominal value; the threshold is chosen to be $1 \%$. The reduced model can be validated if the voltage and current values at the electric lines do not exceed the physical allowed limits.

For the district heating network, the global level corresponds to the heating source, and the local level is at the substations. At the heat pump and storage tank level, the power error will be minor when a low-energy building is removed. At the substation's level, there will be a $100 \%$ error of power. In the district heating network, the temperature and the mass flow should be analyzed. The re- duced model's mass flow and temperature should not vary significantly compared to the full model's values. At the substation level of the building removed, the mass flow will be equal to zero because the power is set to zero. The substation's model ensures a minimum mass flow. The temperature $T_{\text {supply }}(t)$ received by a building $i$ may vary when removing a building on the same subnetwork due to losses in the pipes that depend on the mass flow.

\subsection{Replacing buildings with empty buildings models}

As stated in section 3, a low-ranked component could be replaced with different types of component models; different possibilities thus exist for building replacement. When the building's energy contributions are chosen to be eliminated, as done in this study, the active and reactive electrical power values and the heating power values are set to zero. Later on, it can be set to another value. Since the building model comprises an envelope model and an energy-system model, a predefined empty model is created for each.

All subcomponents and internal connections are removed from the envelope and energy-system models; connectors and parameters only remain. The removal of the internal connections decreases the number of equations of the full model. The energy system model's outputs are the active and reactive electrical power values connected to the electrical grid and the heating power value connected to the district heating network. These values are set to zero.

Other surrogate models of buildings can be defined, like an energy-system connected only to the electrical grid or the heating network. Energy systems without PV panels can also be possible. It would be interesting to compare all the results of these possibilities of simplification. This paper's work is limited to replacing the envelope model and the energy-system model with empty models.

\section{Results}

The energy-based ranking method is applied to the district model. The full model does not have to be simulated to obtain the ranking; the simulation of the buildings model is only needed. In the case where the model of the buildings is too large for the solver and cannot be simulated, each building can be simulated separately. In other applications, a limitation of the method can appear when the model cannot be simulated to calculate the activities of the components.

For each building, three types of activities are analyzed: the active electrical activity $A_{P_{\text {total }, \text { elec }}}$, the reactive electrical activity $A_{Q_{\text {total } \text { elec }}}$ and the heating activity $A_{P_{\text {total,heat }}}$. The three activities are obtained by applying the Equation 2 for a one-year interval with $P_{\text {total,elec }(t)}, Q_{\text {total,elec }}(t)$ and $P_{\text {total,heat }}(t)$ considered as the three energy flowing to the building. The horizon could also be chosen to be a single season of interest. However, in this case study, all buildings share the same synthetic consumption scenario. 
Thus the effect of seasonal variations affects activity magnitudes but cancels out in the activity ranking.

The activities are calculated for each of the 20 buildings during the simulation. The ranking of the buildings for each of the three activities is then deduced. The ranking depends on the model, especially on the input scenario and the parameters of the system. As the ranking is roughly the same between the three tables' shared buildings, only the Table 1 from the electrical active power point of view is presented. The buildings that will be simplified are easily identified.

Differences in the ranking of the three activities can happen due to a difference in the scenario between buildings or a change in the time slot. In this case, a suitable replacement building model will have to be found that combines the simplification according to multiple ranking.

A limitation of this method is when a majority of buildings have similar and low activity values. Then, it is not possible to remove low activity buildings while preserving most of the total activity of the system. Aggregation might be an alternative solution to combine buildings with similar properties. When using aggregation, the nodes of each of the district heating network and the electrical grid should be aggregated as well. A work on aggregating the nodes of a Modelica district heating network model can be found in Falay et al. (2020).

Table 1. Ranking of the buildings from the electrical active power point of view

\begin{tabular}{llll}
\hline Buildings & $\begin{array}{l}\text { Activity } \\
{[M W h]}\end{array}$ & $\begin{array}{l}\text { Relative } \\
(\%)\end{array}$ & $\begin{array}{l}\text { Accumulated } \\
(\%)\end{array}$ \\
\hline Building 20 & 2270 & 26 & 26 \\
Building 15 & 1294 & 15 & 41 \\
Building 16 & 890 & 10 & 51 \\
Building 14 & 627 & 7 & 58 \\
Building 12 & 618 & 7 & 65 \\
Building 17 & 612 & 7 & 72 \\
Building 13 & 526 & 6 & 78 \\
Building 03 & 400 & 5 & 82 \\
Building 06 & 325 & 4 & 86 \\
Building 11 & 260 & 3 & 89 \\
Building 18 & 223 & 3 & 91 \\
Building 05 & 197 & 2 & 94 \\
Building 09 & 151 & 2 & 95 \\
Building 10 & 143 & 2 & 97 \\
Building 04 & 95 & 1 & 98 \\
Building 19 & 77 & 1 & 99 \\
Building 08 & 51 & 1 & 100 \\
Building 07 & 14 & 0 & 100 \\
Building 02 & 14 & 0 & 100 \\
Building 01 & 13 & 0 & 100 \\
Total & 8800 & & \\
\hline
\end{tabular}

\subsection{Simulation time}

The multi-energy model, shown in Figure 1, is simulated for a one-year period with one-hour sampled inputs using CVODE solver with a variable step. The building model interacts with the electrical grid and district heating network models; this interaction causes higher simulation time. The simulation time is expected to decrease when buildings are simplified. The number of equations decreases linearly as in Figure 5 with the decrease of the number of buildings to simulate. The simulation time does not follow a linear decrease as shown in Figure 6. For example, simplifying the four low-ranked buildings leaves 16 buildings to simulate. The full model's simulation time is thus reduced by almost a factor of two.

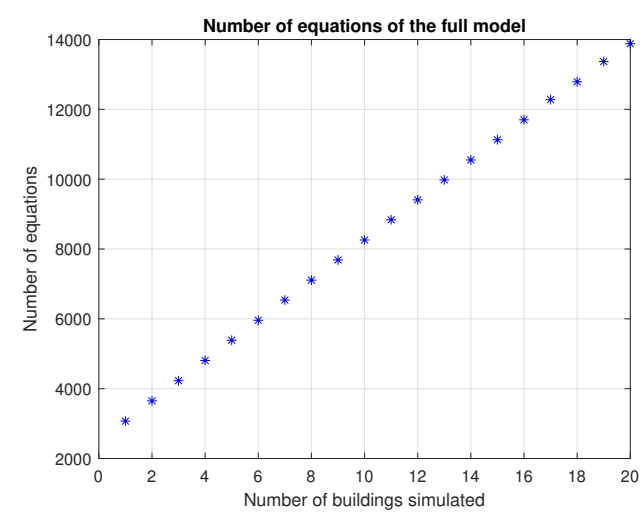

Figure 5. Number of equations of the full model

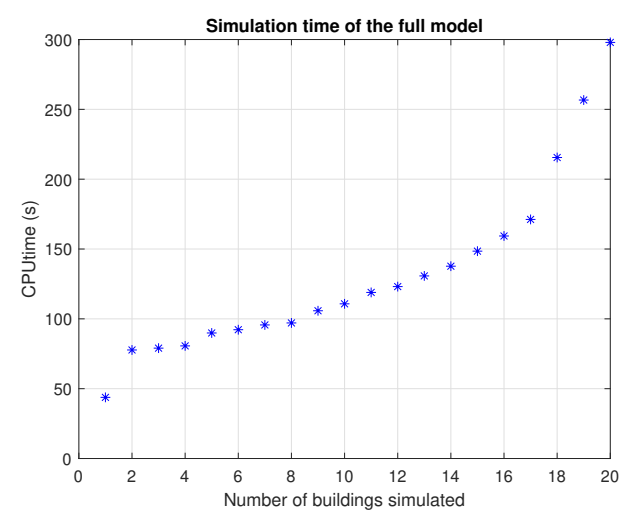

Figure 6. Simulation time of the full model

\subsection{Reduced model verification}

After the reduction is applied, the accuracy of the city district reduced model should be verified. Criteria for several signals are defined to calculate the error generated by the simplification of the buildings. The red indices in the criteria definitions correspond to the values of the signals obtained with the reduced model's simulation.

Criteria are introduced for the electrical grid verification in Equation 3, Equation 4 and Equation 5 with $I(t), U(t)$ the values of the current and voltage at a line obtained with 
the full model's simulation. $U_{\text {nom }}$ is the nominal value of the voltage at a line. Equation 4 represents the mean absolute error (MAE) of the current relative to the current's maximum value. $T$ is the time span of the signal values. Equation 6 and Equation 7 are introduced for the heating network with $T_{\text {supply }}(t), \dot{m}(t)$ the values of the received temperature and the mass flow at a substation of a building obtained with the full model's simulation.

$$
\begin{array}{r}
C I_{\infty}(\text { line } i)=\frac{\left\|I(t)-I_{\text {red }}(t)\right\|_{\infty}}{\|I(t)\|_{\infty}} \\
C I_{M A E}(\text { line } i)=\frac{\frac{1}{T} \int_{0}^{T}\left|I(t)-I_{\text {red }}(t)\right|}{\|I(t)\|_{\infty}} \\
C U_{\infty}(\text { line } i)=\frac{\left\|U(t)-U_{\text {red }}(t)\right\|_{\infty}}{U_{\text {nom }}} \\
C T_{\infty}(\text { building } i)=\frac{\left\|T_{\text {supply }}(t)-T_{\text {supply,red }}(t)\right\|_{\infty}}{\left\|T_{\text {supply }}(t)\right\|_{\infty}} \\
C \dot{m}_{\infty}(\text { building } i)=\frac{\left\|\dot{m}(t)-\dot{m}_{\text {red }}(t)\right\|_{\infty}}{\|\dot{m}(t)\|_{\infty}}
\end{array}
$$

The case study is composed of 20 buildings. The four buildings ranked last in the ranking of Table 1 are removed at once, which leaves a model of 16 buildings to simulate. The removal of the contribution of these buildings affects the variables' values of the electrical grid and heating network, which should be validated using the defined criteria. The electrical grid is composed of 23 lines connected to medium and low voltage buildings. After reducing the buildings, the current of these lines does not surpass the maximum value $I_{\max }$ allowed of the line, and the voltages at the lines are far from the limits. Equation 3, Equation 4, and Equation 5 are represented in Figure 7 and Figure 8.

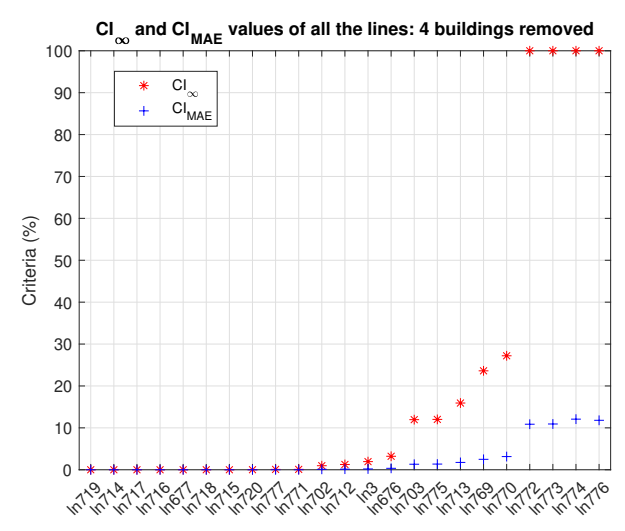

Figure 7. Error on the current of the lines of the electrical grid with respect to Equation 3 and Equation 4

In Figure 7, the error is $100 \%$ for the lines $772,773,774$ et 776 because these lines are directly connected to the four removed buildings (see Figure 3). For the other lines, the error on the current does not exceed $30 \%$ of the maximum value of the current with the full model's simulation, and the MAE criterion values are small. These values are acceptable since the current values of the reduced model are

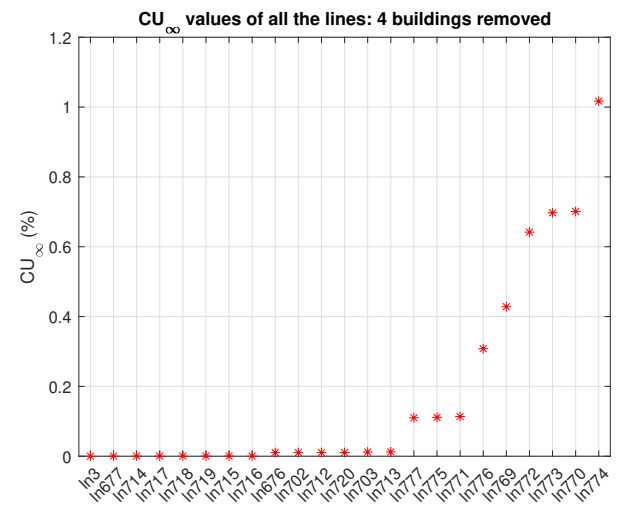

Figure 8. Error on the voltage of the lines of the electrical grid with respect to Equation 5

far from the $I_{\max }$ limits. In Figure 8 , the criteria on the voltage values do not exceed $1 \%$ of the nominal voltage value of the line, except for line 774 , where the building is removed. Voltages are less sensitive than the currents to the variation of the power values of the buildings. The error on the lines depends on the position of the line relative to the building removed. In Figure 9, the different criteria values are presented for line 3 at the source level with respect to the number of buildings simulated. As expected, the current criteria values increase when more buildings are removed. The voltage at the line 3 does not vary significantly.

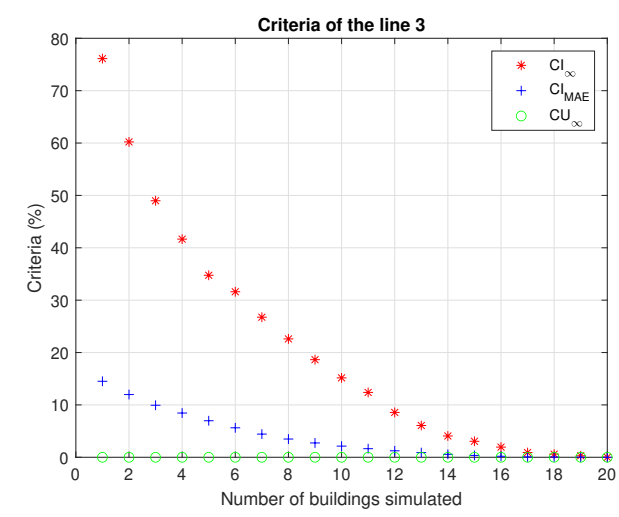

Figure 9. Criteria of the line 3 while removing buildings

The district heating network is affected when removing buildings. The power demand is set to zero; this sets the building's mass flow $\dot{m}_{\text {red }}$ of the reduced model to zero. Building 1,2, 7, and 8 are the four removed buildings, and they have an error of mass flow of $100 \%$ between the full and reduced model, not shown in Figure 10. The other buildings are negligibly affected depending on their positions relative to the removed buildings (see Figure 4). In Figure 11, buildings on the same subnetwork have the same temperature error; this is because of the way of modeling the losses in the pipes in the district heating network. Buildings on subnetworks that are not affected have a zero 


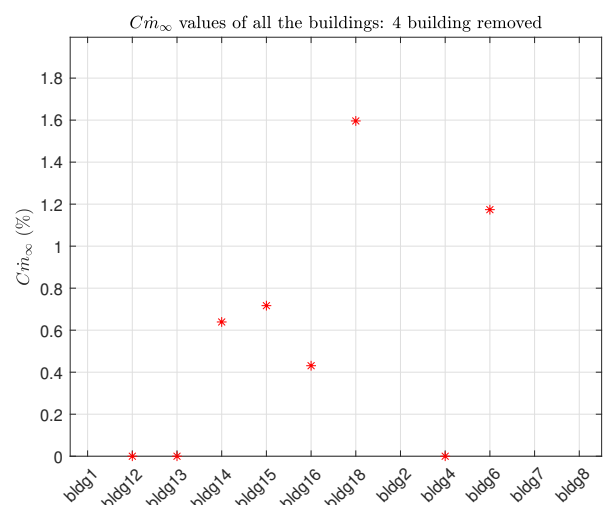

Figure 10. Error on the demanded mass flow of the buildings of the district heating network with respect to Equation 7

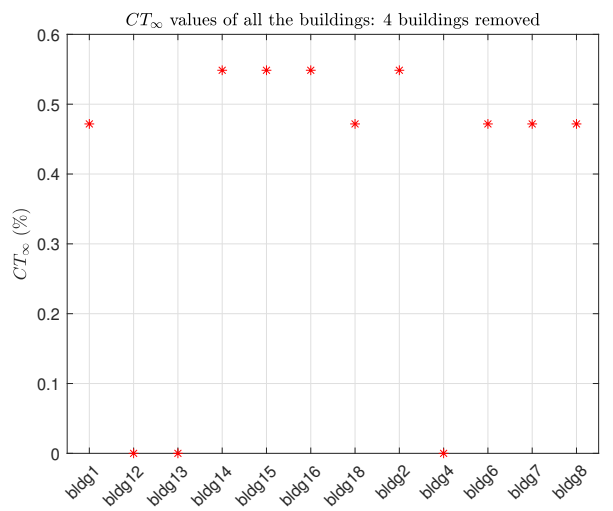

Figure 11. Error on the received temperature of the buildings of the district heating network with respect to Equation 6

error. In Figure 12, the different criteria values are presented at the heating source level for the number of buildings simulated. The error is expected to increase when buildings are simplified. However, mass flow error values are high when simulating 5,7 , and 8 buildings. No explanation is yet certain, but this result is affected by the subnetwork assigned to the removed buildings, where there are heat losses.

An energy criterion at line 3 is defined in Equation 8, where $\sum_{k}\left|P_{\text {total,elec }}(k)\right|$ is the sum of one-hour samples over a one year simulation (8761 samples). The results in Figure 13 reflect the error on the demanded power, caused by the removed building's contribution from Table 1.

$$
\text { CE }(\text { line } 3)=\frac{\left|\sum_{k}\right| P_{\text {total }, \text { elec }}(k)\left|-\sum_{k}\right| P_{\text {total }, \text { elec, }, \text { red }}(k)||}{\sum_{k}\left|P_{\text {total }, \text { elec }}(k)\right|}
$$

\section{Conclusion}

In this paper, a proof of concept for the method of reducing complex multi-energy models is presented. This method conserves the model's physical meaning by reducing the model's components using energy-based ranking. Components are removed or replaced with a simpler model

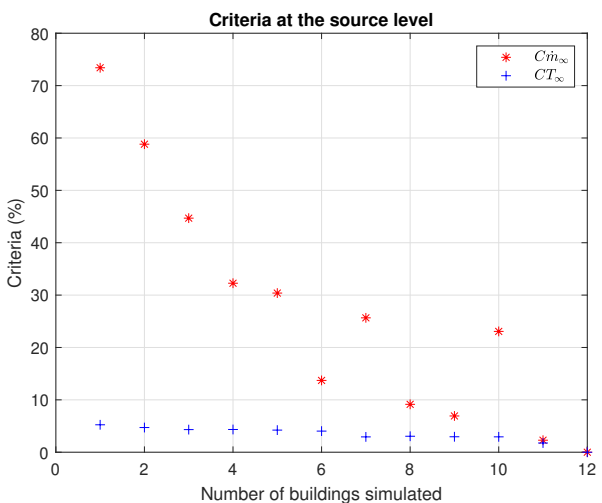

Figure 12. Criteria at the source level of the district heating network while removing buildings

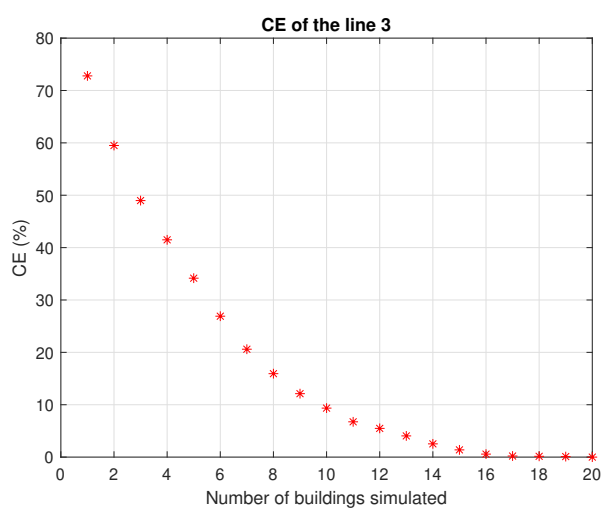

Figure 13. Error on the energy at the electric source level

using the replaceable and redeclare properties. The reduced model has a shorter simulation time, the precision is evaluated by the criteria proposed, and the physical constraints were respected. It is interesting for future work to find a suitable number for removed buildings by compromising between the simulation time and the model's precision. An estimation of the local error at the electrical grid and heating network levels is practical and replaces simulating the reduced model and the full model to compare them. As a next step, we will test our method on a larger scale model and on a different time horizon and inputs. For cases when this ranking-based model reduction doesn't apply well (e.g. when a large fraction of components share similar low activity values), a to-be-defined component aggregation strategy would be complementary.

\section{Acknowledgements}

This work has been supported by Électricité de France (EDF R\&D) and RISEGrid Institute. This work reuses parts of the PowerGrid demonstrator which was partly funded and supported by BPI France through the French FUI ModeliScale research project. The authors would like to thank Jean-Philippe Tavella, Dominique Croteau, Hassan Bouia, and Clément Flinois for their support with the model implementation and expertise in the various fields. 


\section{References}

Bouquerel, Mathias et al. (2019). "Requirements modelling to help decision makers to efficiently renovate energy systems of urban districts". In: Proceedings of the 2019 Summer Simulation Conference, pp. 1-12.

Deng, Kun et al. (2014). "Structure-preserving model reduction of nonlinear building thermal models". In: Automatica 50.4, pp. 1188-1195.

Falay, Basak et al. (2020). "Enabling large-scale dynamic simulations and reducing model complexity of district heating and cooling systems by aggregation". In: Energy 209, p. 118410.

Fritzson, Peter and Vadim Engelson (1998). "Modelica-A unified object-oriented language for system modeling and simulation". In: European Conference on Object-Oriented Programming. Springer, pp. 67-90.

Kim, Eui-Jong et al. (2014). "Urban energy simulation: Simplification and reduction of building envelope models". In: Energy and Buildings 84, pp. 193-202.

Louca, Loucas Sotiri (1998). "An energy-based model reduction methodology for automated modeling." $\mathrm{PhD}$ thesis.

Marshall, SA (1966). "An approximate method for reducing the order of a linear system". In: Control 10, pp. 642-653.

Moore, Bruce (1981). "Principal component analysis in linear systems: Controllability, observability, and model reduction". In: IEEE transactions on automatic control 26.1, pp. 17-32.

Plessis, Gilles, Aurelie Kaemmerlen, and Amy Lindsay (2014). "BuildSysPro: a Modelica library for modelling buildings and energy systems". In: Proceedings of the 10 th International Modelica Conference; March 10-12; 2014; Lund; Sweden. 096. Linköping University Electronic Press, pp. 1161-1169.

Sodja, Anton, Igor Škrjanc, and Borut Zupančič (2019). "Realization-preserving model reduction of object-oriented models using energy-based metrics". In: Simulation 95.7, pp. 607-620.

Sodja, Anton, Igor Škrjanc, and Borut Zupančič (2020). “Cyberphysical modelling in Modelica with model-reduction techniques". In: Journal of Systems and Software 163, p. 110517.

Tavella, Jean-Philippe (2020). PowerSysPro library. URL: https: //bitbucket.org/simulage/powersyspro/wiki.

Tiller, Michael (2014). Modelica by Example. URL: https://mbe. modelica.university/components/architectures/replaceable/. 